\title{
Precision Model for Microwave Rotary Vane Attenuator
}

\section{Guldbrandsen, Tom}

\section{Published in:}

I E E E Transactions on Instrumentation and Measurement

Link to article, DOI:

10.1109/TIM.1979.4314761

Publication date:

1979

Document Version

Publisher's PDF, also known as Version of record

Link back to DTU Orbit

Citation (APA):

Guldbrandsen, T. (1979). Precision Model for Microwave Rotary Vane Attenuator. I E E E Transactions on Instrumentation and Measurement, 28(1), 59-66. https://doi.org/10.1109/TIM.1979.4314761

\section{General rights}

Copyright and moral rights for the publications made accessible in the public portal are retained by the authors and/or other copyright owners and it is a condition of accessing publications that users recognise and abide by the legal requirements associated with these rights.

- Users may download and print one copy of any publication from the public portal for the purpose of private study or research.

- You may not further distribute the material or use it for any profit-making activity or commercial gain

- You may freely distribute the URL identifying the publication in the public portal

If you believe that this document breaches copyright please contact us providing details, and we will remove access to the work immediately and investigate your claim. 


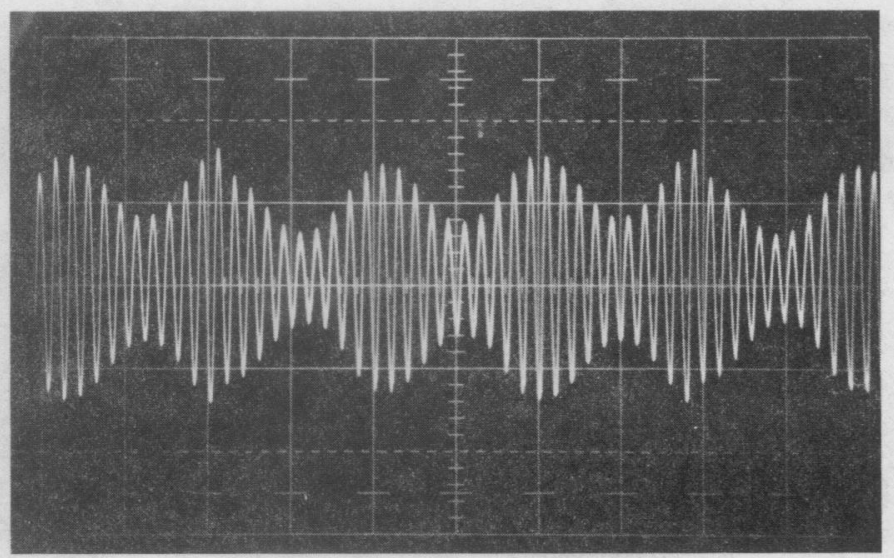

Fig. 10. Amplitude modulated carrier. Carrier $=1 \mathrm{kHz}$. Modulating signal $=100 \mathrm{~Hz}$. Vertical scale $=2 \mathrm{~V} /$ Div

been used. However, a radio-frequency carrier can be used easily by replacing the $1-\mathrm{kHz}$ oscillator with a radiofrequency oscillator and by redesigning the peak detector only. The rest of the circuits will remain the same since the signals at these stages are of low (modulating) frequency.

\section{CONCLUSION}

A simple feedback scheme for controlling the amplitude of oscillators by means of a voltage has been described. The control is precise and linear. Two applications of the scheme have been considered; one in amplitude stabilization of oscillators and the other in generation of stabilized ampli- tude modulation. The experimental results agree closely with theoretical predictions.

In the examples discussed in the paper, the scheme stabilized the amplitude of oscillation against variations with respect to frequency. However the stabilization will remain effective against variation due to other parameters such as temperature, loading, etc. It should also be noted that the voltages $V_{R 1}, V_{R 2}$, and the gain $A$ are the only parameters that may need adjustment as we move from one oscillator to another. Thus by providing terminals for $V_{R 1}$ and $V_{R 2}$ and a potentiometer control for $A$, the feedback part of the circuit can be mass produced and easily adapted to different oscillators. Finally, the scheme, though presented for sinusoidal oscillators, should be applicable for other types such as square wave oscillators as well.

\section{REFERENCES}

[1] D. Roddy and J. Coolen, Electronic Communications. Englewood Cliffs, NJ: Prentice-Hall, 1977.

[2] W. D. Cooper, Electronic Instrumentation and Measurement Techniques. Englewood Cliffs, NJ: Prentice-Hall, 1970.

[3] F. E. Terman and J. M. Pettit, Electronic Measurements. New York: McGraw-Hill, 1952.

[4] Instruction Manual, HF Generator PM 5324, Philips, 1973.

[5] N. H. Crowhurst, Basic Oscillator Handbook. New York: Gernsback Library, 1966.

[6] A. Budak, Passive and Active Network Analysis and Synthesis. Boston, MA: Houghton Mifflin, 1974.

[7] J. G. Graeme, Designing with Operational Amplifiers. New York: McGraw-Hill, 1977.

[8] M. Sundaramurthy, B. B. Bhattacharyya, and M. N. S. Swamy, "A simple voltage controlled oscillator with grounded capacitors," Proc. IEEE, vol. 65, pp. 1612-1614, Nov. 1977.

\title{
Precision Model for Microwave Rotary Vane Attenuator
}

\author{
TOM GULDBRANDSEN
}

\begin{abstract}
A model for a rotary vane attenuator is developed to describe the attenuator reflection and transmission coefficients in detail. All the parameters of the model can be measured in situ, i.e., without disassembling any part. The transmission errors caused by internal reflections are calculated from measurements of the much larger reflection parameters, hence commonly used nonprecision instruments can be used to determine the transmission errors with sufficient accuracy for the highest precision obtainable in standard laboratories.
\end{abstract}

Manuscript received April 18, 1978.

The author is with Physics Laboratory III, Technical University of Denmark, DK-2800 Lyngby, Denmark.

\section{INTRODUCTION}

$\Gamma^{T}$ IS WELL KNOWN that rotary vane attenuators (abbreviated RVA) are widely used for microwave attenuation measurements. This is due to the direct and accurate reading of the attenuation and to the rather constant (attenuation independent) phase shift.

Commercially available RVA's typically have an accuracy of $\pm(0.1 \mathrm{~dB}+1$ percent of decibel reading $)$ and $\pm 2^{\circ}$ phase change up to $50 \mathrm{~dB}$. Although this is sufficient for many purposes, some standard laboratory measurements require much higher precision. 
RVA's have been built with much higher precision [1]-[5]. With such attenuators, deviations from the ideal attenuation versus vane-angle relationship can be kept below $0.01 \mathrm{~dB}$ for attenuations up to more than $40 \mathrm{~dB}$. Although this is much better than the accuracy of commercial attenuators it is still far from taking full advantage of the stability of the setup and of the resolution and accuracy of the direct angle readout systems used. Furthermore, the variation of phase angle is not negligible, being typically $0.5^{\circ}$ from 0 to $40 \mathrm{~dB}$, which corresponds to roughly 0.1 "imaginary decibels." Most of the errors can, in principle, be reduced by more careful fabrication in order to avoid internal reflections, leakage, etc., but the above mentioned RVA's probably represent the state of the art. The RVA can be calibrated as far as attenuation is concerned, for instance, by means of the dc substitution or the modulated subcarrier technique. This is described in [4] where a considerably higher precision is obtained for the calibrated attenuator.

In this paper a complete precision model for the RVA is developed. All the parameters of the model can be measured with sufficient accuracy by means of ordinary nonprecision components or instruments, and all measurements can be performed in situ, i.e., without disconnecting any part. (The attenuator may, for instance, be part of an interferometer.) In addition, the parameters are independent of external circuits and the RVA can, therefore, be transported between laboratories without risk of parameter change due to imperfect tuning. By using this model with the measured parameters, a precision can be attained which is limited only by noise, short-term drift, and the repeatability of switches.

The principles are illustrated by measurements of the parameters for a commercial $X$-band RVA (HewlettPackard HP X382A). The principles may be used in the frequency range from $S$ - to $G$-band $(2.6-220 \mathrm{GHz})$ where RVA's and the other components used (switches and circulators) are commercially available.

\section{MOdel for a Precision Rotary VANE ATTENUATOR}

It is assumed that higher order modes, readout errors, and mechanical errors (runout and play) are negligible. Errors from reflections between the RVA and other components in the system are avoided by isolating the RVA by means of two circulators.

\section{A. Model for RVA Without Circulators}

Calculations of this type have been given earlier in less detail by Hand [6], Holm et al. [7], and Otoshi and Stelzried [8]. Fig. 1 shows a sketch of the attenuator sections. The scattering matrix of the RVA is calculated by combining the matrices of the three sections. The calculation is performed by means of a series development which converges rapidly, because deviations from the ideal matrices are small. The calculations are performed in detail in Appendix I.

The angular dependence of the elements in the scattering matrix resulting from these calculations is given by

1) Reflection at port $R_{1}$

$$
\begin{aligned}
\rho_{R}= & a_{R}+b_{R} \sin ^{2} \theta+c_{R} \sin ^{2} 2 \theta \\
& +d_{R} \sin 2 \theta+e_{R} \sin 4 \theta .
\end{aligned}
$$

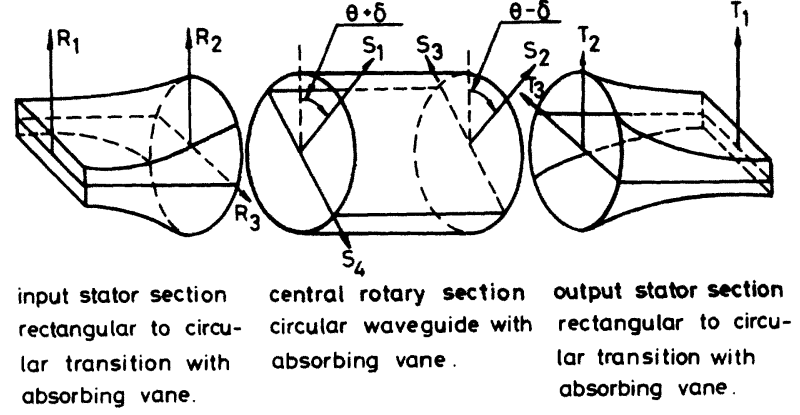

Fig. 1. Attenuator sections with definitions of ports and rotation angles. Directions of coordinate axes are chosen parallel to principal axes of $\mathrm{TE}_{11}$ modes in circular guides.

2) Reflection at port $T_{1}$

$$
\begin{aligned}
\rho_{T}= & a_{T}+b_{T} \sin ^{2} \theta+c_{T} \sin ^{2} 2 \theta \\
& +d_{T} \sin 2 \theta+e_{T} \sin 4 \theta .
\end{aligned}
$$

3) Transmission between port $R_{1}$ and port $T_{1}$

$$
\begin{aligned}
T= & T_{0} \cos ^{2} \theta\left(1+\alpha \sin ^{2} \theta+\beta \sin ^{2} 2 \theta+j \varepsilon \tan \theta\right. \\
& \left.+\gamma \tan ^{2} \theta+\zeta \sin 2 \theta+\eta \sin 4 \theta\right)
\end{aligned}
$$

where the parameters in the above expressions are functions of the elements in the matrices in Figs. 10 and 11. These functions are given in Appendix I.

From the functions one finds the following important relations between the transmission parameters $\alpha, \beta, \zeta$, and $\eta$ and the first-order terms in the reflection parameters:

$$
\begin{aligned}
\alpha= & -\frac{4}{T_{0}^{2}}\left(b_{R} c_{T}+b_{T} c_{R}-4 c_{R} c_{T}\right) \\
\beta= & -\frac{4}{T_{0}^{2}} c_{R} c_{T} \\
\zeta= & -\frac{4}{T_{0}^{2}}\left[+d_{R} c_{T}+d_{T} c_{R}+e_{R} b_{T}\right. \\
& \left.+e_{T} b_{R}-4\left(e_{R} c_{T}+e_{T} c_{R}\right)\right] \\
\eta= & -\frac{4}{T_{0}^{2}}\left(e_{R} c_{T}+e_{T} c_{R}\right) .
\end{aligned}
$$

These relations show that the small second-order transmission error parameters $\alpha, \beta, \zeta$, and $\eta$ can be determined from measurements of the much larger and, therefore, more easily measurable reflection parameters.

\section{B. Model for RVA with Circulators}

The RVA is modified by means of two circulators and two switches as shown in the scattering diagram of Fig. 2. The circulators isolate the RVA from the outer circuit and thereby make the incremental attenuation and phase shift virtually independent of the outer circuit. The four switch positions enable forward and reverse transmission through the RVA, and input and output reflection to be measured in situ. By means of the scattering diagram for the modified RVA one may show that the forms of the transmissions and reflections are unchanged relative to the RVA without 


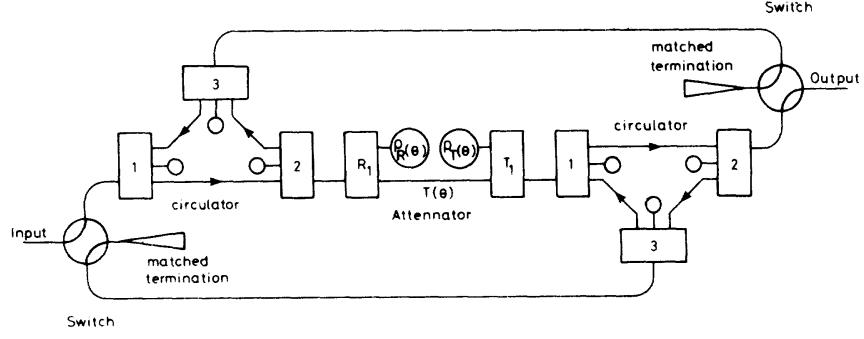

Fig. 2. Scattering diagram for RVA modified with circulators and switches.

circulators. The transmission coefficients of the modified RVA are dependent on the transmission direction due to the circulators, i.e., $T_{0}$ is substituted by $T_{F}$ and $T_{R}$ for forward and reverse transmission whereas $\alpha, \beta, \varepsilon, \gamma, \delta, \zeta$, and $\eta$ are still independent of transmission direction. It is also seen from the scattering diagram that the important relations (1)-(4) apply for the modified RVA if $T_{0}^{2}$ is substituted by $T_{F} \cdot T_{R}$. Finally, the orders of magnitude of all the parameters are unchanged.

\section{Procedures for Determination of Model Parameters}

\section{A. Determination of $\alpha$ and $\beta$}

The reflection parameters $b_{R}, b_{T}, c_{R}$, and $c_{T}$ are ordinarily about 0.05 and give rise to $\alpha$ and $\beta$ values of the order of $10^{-3}$.

The best stability obtained in attenuation measurements (in national standard laboratories) is of the order of $10^{-5}$ (or $10^{-4} \mathrm{~dB}$ ) at low attenuations. In order to reduce the attenuation errors below this limit, the reflection parameters should be measured with an accuracy of about $10^{-4}$. The transmission parameters $T_{F}$ and $T_{R}$ are of the order of 1 and should be measured with an accuracy of $10^{-2}$.

The accuracy required for the reflection parameters $b_{R}$, $b_{T}, c_{R}$, and $c_{T}$ is rather easily obtained due to two important facts:

1) $b_{R}, b_{T}, c_{R}$, and $c_{T}$ are all incremental reflection coefficients, i.e., differences between reflection coefficients measured at different angles of the RVA.

2) An arbitrary complex factor inserted anywhere outside the circulators will enter the expressions for $b_{R}, b_{T}, c_{R}, c_{T}, T_{F}$, and $T_{R}$ in such a way that it cancels out in the expressions for $\alpha$ and $\beta$.

This means that the well-known problem of accurate measurements of reflection phase as well as amplitude has been solved for this specific application without the need for disassembling and reassembling any part.

The reflection parameters are easily measured with sufficient accuracy if the RVA is part of an interferometer. This is especially convenient because the measurements may be performed immediately before and/or after precision transmission measurements in order to avoid temperature drift of $\alpha$ and $\beta$. Nonprecision network analyzers may in many cases be used because of the conditions 1) and 2).

$b_{R}$ and $c_{R}$ are determined from a Fourier transform of the reflection $\rho_{R}(\theta)$ from port $R_{1}$. In most cases, they can be determined with sufficient accuracy from measurements at angles $\theta=0^{\circ}, 45^{\circ}, 90^{\circ}$, and $135^{\circ}$ by the expressions

$$
\begin{aligned}
& b_{R}=\rho_{R}\left(90^{\circ}\right)-\rho_{R}\left(0^{\circ}\right) \\
& c_{R}=\frac{1}{2}\left[\rho_{R}\left(45^{\circ}\right)+\rho_{R}\left(135^{\circ}\right)-\rho_{R}\left(0^{\circ}\right)-\rho_{R}\left(90^{\circ}\right)\right]
\end{aligned}
$$

and similarly for $b_{T}$ and $c_{T}$.

\section{B. Direct Determination of $\alpha$ and $\beta$ from Precision Transmission Measurements}

Direct determination requires high precision transmission measurements and we are not yet able to perform such measurements due to poor accuracy of the angle readout. The present readout error of $0.2^{\circ}$ is going to be reduced considerably by means of an optoelectronic angle encoder mounted directly onto the rotary section.

In Section IV an analysis is given of the attenuation measurements presented in [3].

\section{Determination of $\zeta$ and $\eta$}

$\zeta$ and especially $\eta$ are very small and often near the detection limit. Using (3) and (4) they may easily be determined with sufficient accuracy with a network analyzer.

\section{Determination of $\varepsilon$, Zero Setting Error $\theta_{0}$, and $\gamma$}

$\varepsilon, \theta_{0}$, and $\gamma$ are not related to the reflection parameters and must be determined directly from measurements of complex transmission. As their relative significance is highest at high attenuation settings they are preferably determined from measurements in the vicinity of $90^{\circ}$. If the transmission measurements are performed at angles $\theta_{1}=90^{\circ}-\theta_{0}$, $\theta_{2}=90^{\circ}-\theta_{0}-\Delta \theta$, and $\theta_{3}=90^{\circ}-\theta_{0}+\Delta \theta$ where $\theta_{1}$ is a first estimate of $90^{\circ}$ and $\left|\theta_{0}\right| \ll \Delta \theta \ll 45^{\circ}$ one obtains the following relations neglecting $\alpha, \beta, \zeta$, and $\eta$ :

$$
\begin{aligned}
t_{1}= & T_{0}\left[\cos ^{2}\left(90^{\circ}-\theta_{0}\right)+\gamma\right]=T_{0}\left(\sin ^{2} \theta_{0}+\gamma\right) \\
t_{2}= & T_{0}\left[\cos ^{2}\left(90^{\circ}-\theta_{0}-\Delta \theta\right)\right. \\
& \left.+j \varepsilon \sin \left(90^{\circ}-\theta_{0}-\Delta \theta\right) \cos \left(90^{\circ}-\theta_{0}-\Delta \theta\right)+\gamma\right] \\
\simeq & T_{0}\left[\sin ^{2} \Delta \theta+\left(2 \theta_{0}+j \varepsilon\right) \sin \Delta \theta \cos \Delta \theta+\gamma\right] \\
t_{3} \simeq & T_{0}\left[\sin ^{2} \Delta \theta-\left(2 \theta_{0}+j \varepsilon\right) \sin \Delta \theta \cos \Delta \theta+\gamma\right] .
\end{aligned}
$$

Solution of the relations with respect to $2 \theta_{0}+j \varepsilon, \gamma$, and $T_{0}$ yields

$$
\begin{aligned}
2 \theta_{0}+j \varepsilon & =\frac{t_{2}-t_{3}}{t_{2}+t_{3}-2 t_{1}} \tan \Delta \theta \\
\gamma & =\frac{2 t_{1} \sin ^{2} \Delta \theta}{t_{2}+t_{3}-2 t_{1}}-\sin ^{2} \theta_{0} \\
T_{0} & =\frac{t_{2}+t_{3}-2 t_{1}}{2 \sin ^{2} \Delta \theta} .
\end{aligned}
$$

The last equation can be used for checking the accuracy of the measurements as $T_{0}$ is already known. The choice of $\Delta \theta$ is a compromise between a high signal level for $t_{2}-t_{3}$ requiring a high $\Delta \theta$ value and a large relative difference between $t_{2}$ and $t_{3}$ requiring a small $\Delta \theta$. The measurements may in many cases be carried out with sufficient accuracy by 


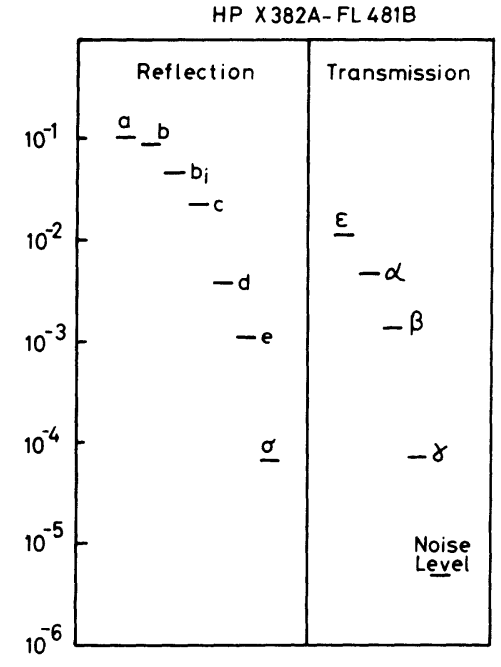

Fig. 3. Maximum moduli of reflection and transmission parameters for the wide-band set of measurements.

means of a network analyzer yielding a few-percent error on the small parameters $\varepsilon$ and $\gamma$.

It should be mentioned that the ordinary method of determination of $\theta_{0}$ from measurements at two angles of equal attenuation (without phase information) in the vicinity of $90^{\circ}$ is in error due to the combined effect of $\gamma$ and $\varepsilon$. If the difference between the two angles is called $2 \Delta \theta$, the error in $\theta_{0}$ (measured minus true) is

$$
\Delta\left(\theta_{0}\right)=\frac{1}{2} \varepsilon \operatorname{Im}(\gamma)(\Delta \theta)^{-2}
$$

and causes a relative transmission error:

$$
\Delta T / T=2 \Delta\left(\theta_{0}\right) \tan \theta
$$

\section{Measurements}

Most of the results presented in this paper originate from one commercial attenuator Hewlett-Packard HP X382A-FL481B which has been improved by means of an angular scale mounted on the worm gear. The readout resolution is $0.01^{\circ}$ measured on the rotary section but the excentricity error is of the order of $0.2^{\circ}$. In order to check the generality of the results, a few measurements have been made on another HP X382A and on a $K_{a}$-band attenuator TRG A510 with similar results. Finally, an analysis has been made of some measurements by F. L. Warner et al. [3].

In order to get both a general view of the magnitude of the parameters and a detailed look at the strongly frequencydependent parameters the measurements on HPX 382A have been performed at two sets of frequencies.

1) Wide-band set: $8.0,8.5, \cdots, 12.0,12.4 \mathrm{GHz}$.

2) Narrow-band set: $9.40,9.42, \cdots, 9.60 \mathrm{GHz}$.

\section{A. Measurements of Reflection Parameters}

For each frequency, the reflected signals from both ends of the RVA are measured at angles $\theta=0^{\circ}, 15^{\circ}, \cdots, 165^{\circ}$. Forward and reverse transmitted signals are measured at $0^{\circ}$ and all reflected signals are normalized by the geometrical mean of the transmitted signals. From a Fourier transform

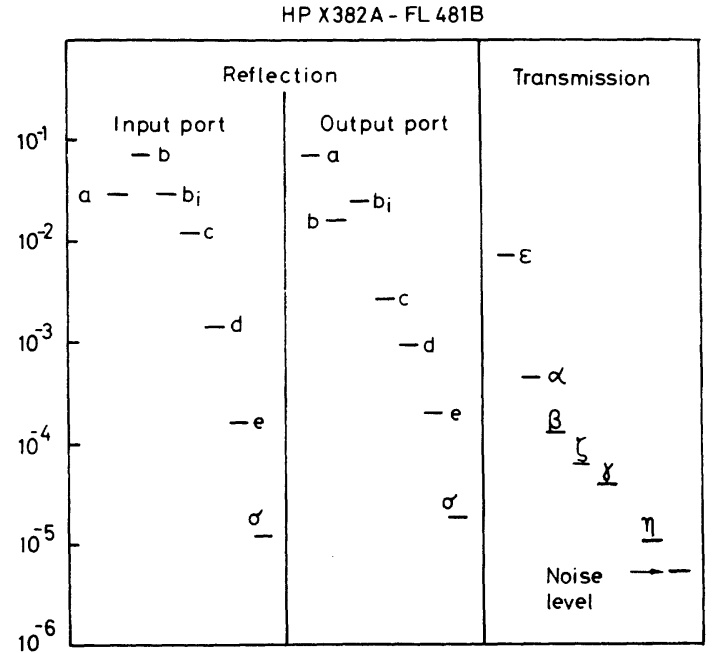

Fig. 4. Moduli of reflection and transmission parameters at $10 \mathrm{GHz}$.

of the normalized reflections versus angle, the parameters $a$, $b, c, d, e$, and seven higher harmonics are calculated for both ends of the RVA. The seven higher harmonics are used to calculate the uncertainty $\sigma$ on a single Fourier component. Fig. 3 shows the highest values of the moduli, $a, b, c, d, e$, and $\sigma$ for the wide-band set of measurements. An additional parameter $b_{i}=-4 c$ is also indicated. Apart from a phase factor, $b_{i}$ is an intrinsic parameter for the RVA, i.e., it is independent of the external components. The small value of $\sigma$ means that the model explains very well the form of the reflections versus vane angle. Fig. 4 shows a plot of the parameters at $10 \mathrm{GHz}$. From the measured reflections, the transmission error parameters $\alpha, \beta, \zeta$, and $\eta$ are calculated. These are also presented in Figs. 3 and 4. $\alpha$ and $\beta$ are strongly frequency-dependent due to the several wavelengths distance between the reflections responsible for $\alpha$ and $\beta$. In order to give an impression of the frequency dependence $\alpha$ and $\beta$ are shown in polar plots (Figs. 5 and 6) made from the narrow-band set of measurements.

\section{B. Measurements of Transmission Parameters}

As mentioned in Section III, the readout accuracy of the RVA used for the measurements is too poor to enable direct determination of $\alpha$ and $\beta$. In order to test the model, an analysis is made of the attenuation measurements presented by Warner et al. in [3, Table I].

The results are corrected for $-104-\mathrm{dB}$ "antiphase leakage" $(\operatorname{Re} \gamma$ in this paper) and for cyclic angular error due to their readout. In order to separate $\operatorname{Re}(\alpha)$ and $\operatorname{Re}(\beta)$ contributions to the attenuation the parameter $\left(\Delta \alpha / \sin ^{2} \theta\right)$ is plotted versus $\sin ^{2} \theta$ in Fig. 7. ( $\Delta \alpha$ is the attenuation error in decibels taken from [3] and should not be confused with the transmission parameter $\alpha$ in the present paper.) This plot is a straight line if the model is correct. From the best fitted straight line $\operatorname{Re}(\alpha)$ and $\operatorname{Re}(\beta)$ can be calculated as shown in the figure. The nice fit indicates that the model is correct and the results $\operatorname{Re}(\alpha)=7 \times 10^{-4}$ and $\operatorname{Re}(\beta)=-4 \times 10^{4}$ seem reasonable taking the VSWR variation from 1.045 to 1.016 into account. 


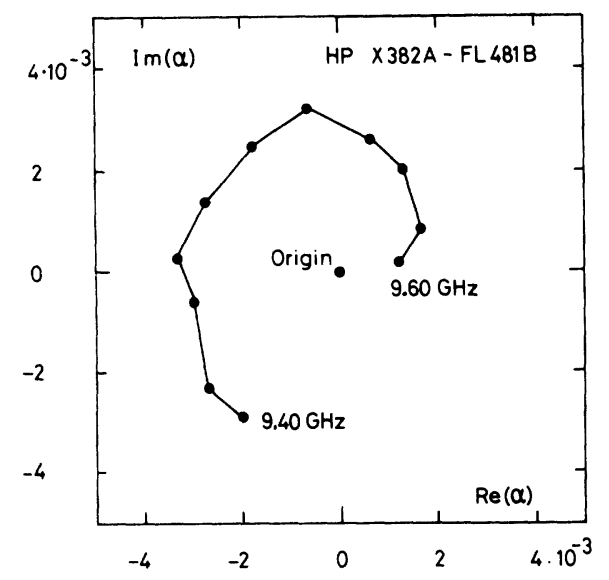

Fig. 5. Polar plot of transmission error parameter $\alpha$ calculated from reflection measurements (narrow-band set). The dot size corresponds roughly to twice the experimental error.

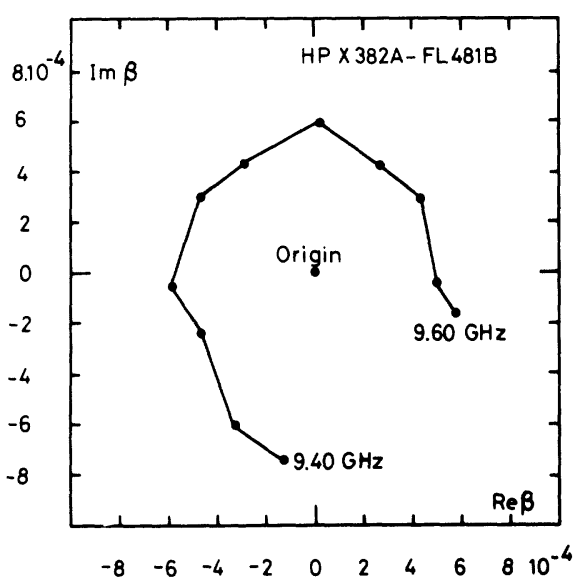

Fig. 6. Polar plot of transmission error parameter $\beta$ calculated from reflection measurements (narrow-band set). The dot size corresponds roughly to the experimental error.

\section{Measurements of $\varepsilon, \theta_{0}$, and $\gamma$}

$\varepsilon$ and $\gamma$ and especially $\theta_{0}$ are slowly dependent on frequency, because no long delay is involved. $\theta_{0}$ is not exactly frequency-independent because the directions of the coordinate systems used for the rotation relations (Appendix I) are chosen along the principal axes of the circular $\mathrm{TE}_{11}$ modes which may differ slightly and frequency dependently from the geometrical axes. $\varepsilon, \theta_{0}$, and $\gamma$ have been measured for the wide-band set of frequencies in the way described in Section III by means of a network analyzer. The measurements are performed both around $90^{\circ}$ and $270^{\circ}$ and polar plots of averages and half differences are shown in Figs. 8(a), (b), and 9(a), (b).

All measurements presented have been taken at $\Delta \theta=1.67^{\circ}$. Measurements taken at other $\Delta \theta$ values show no significant dependence on $\Delta \theta$.

The magnitude of $\gamma$ is rather large and its real part varies considerably with frequency near the band edges. As $\gamma$ is found to be considerably smaller for another HP X382A RVA it seems probable that neither $\delta$ nor $s_{34} / S_{12}$ is responsible for $\gamma$. In general, it is more likely that $\gamma$ for a well

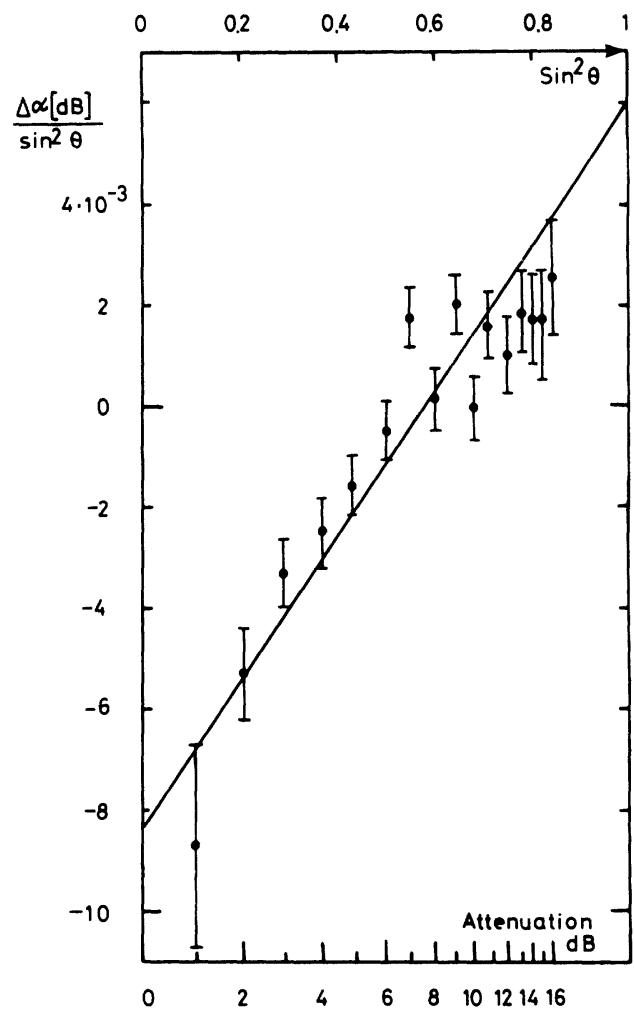

Fig. 7. Plot of attenuation error $\Delta \alpha$ in decibels from [3, Table I]. A straight line with the equation

$$
\frac{\Delta \alpha[\mathrm{dB}]}{\sin ^{2} \theta}=\frac{20}{\ln 10}\left[\operatorname{Re}(\alpha)+4\left(1-\sin ^{2} \theta\right) \operatorname{Re}(\beta)\right]
$$

is fitted to the measured points. Fitted values are $\operatorname{Re}(\alpha)=7 \times 10^{-4}$ and $\operatorname{Re}(\beta)=-4 \times 10^{-4}$. Error bars correspond to the combined effect of $\pm 10^{-3}$ degree angular error and $\pm 2 \times 10^{-4}-\mathrm{dB}$ attenuation error.

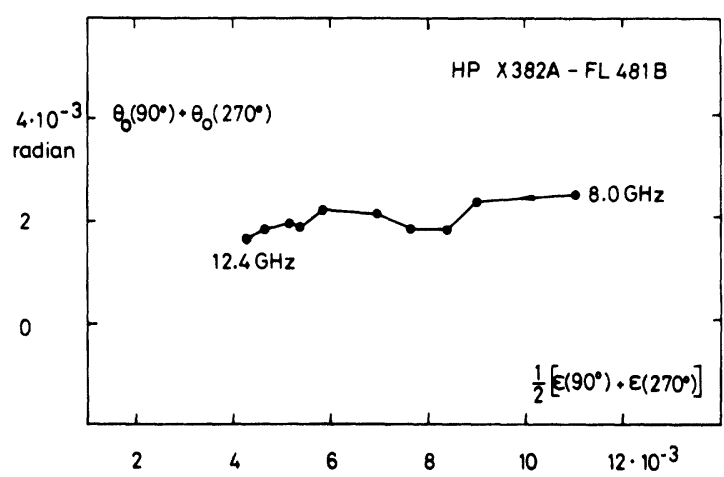

(a)

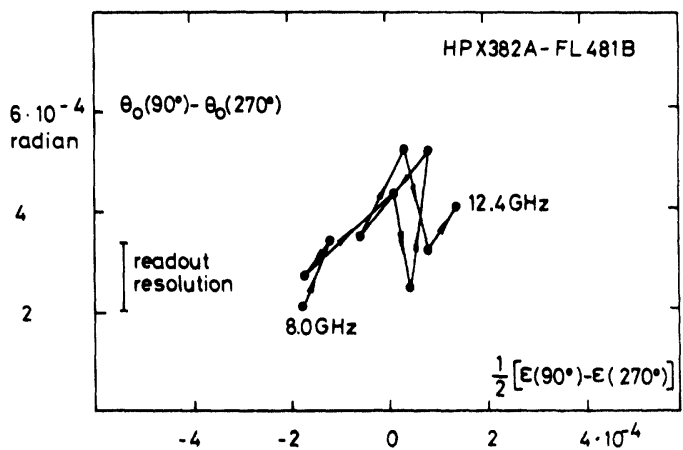

(b)

Fig. 8. Polar plots of $2 \theta_{0}+j \varepsilon$. (a) Average of $90^{\circ}$ and $270^{\circ}$ measurements. The dot size corresponds roughly to the experimental error. (b) Half difference between $90^{\circ}$ and $270^{\circ}$ measurements. 


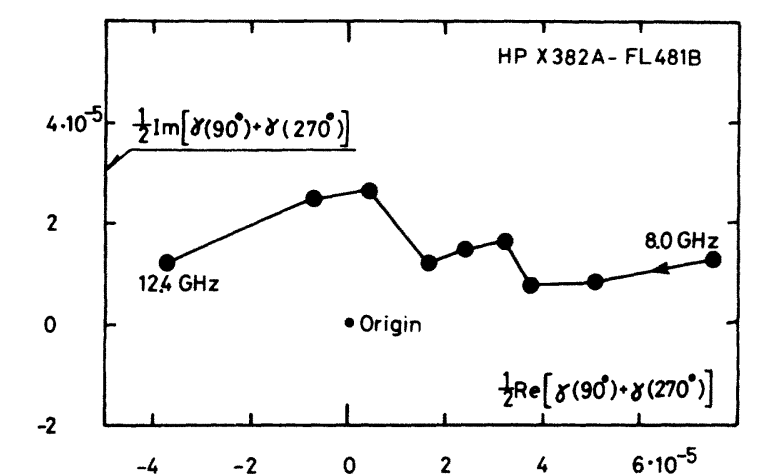

(a)

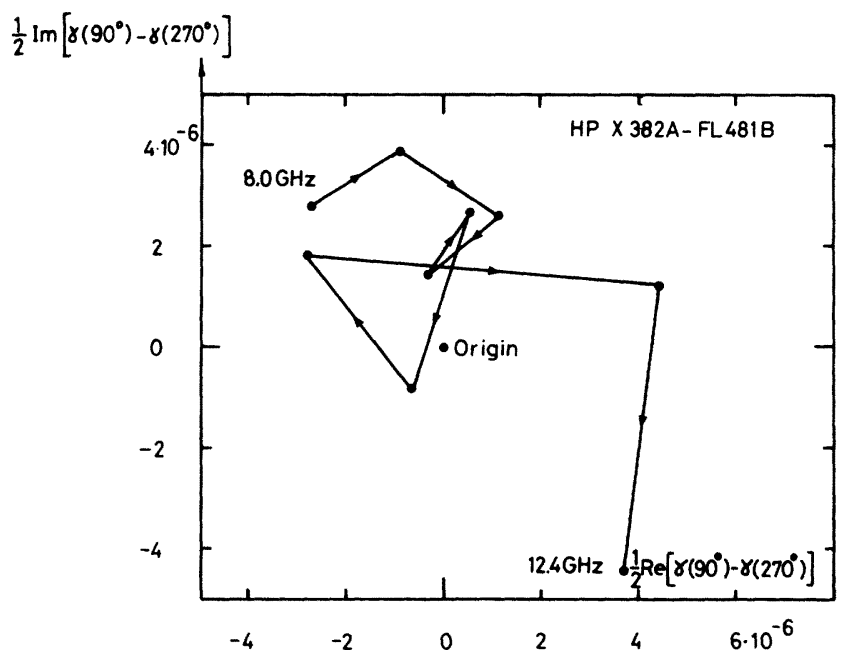

(b)

Fig. 9. Polar plots of $\gamma$. (a) Average of $90^{\circ}$ and $270^{\circ}$ measurements. The dot size corresponds roughly to the experimental error. (b) Half difference between $90^{\circ}$ and $270^{\circ}$ measurements.

designed RVA is dominated by the hitherto disregarded real product terms

$$
\frac{r_{13} S_{13}}{R_{12} S_{12}}+\frac{t_{13} S_{24}}{T_{12} S_{12}}-\frac{r_{13} t_{13}}{R_{12} T_{12}}
$$

This is because the reduction of these terms requires highly rotationally symmetric waveguides whereas $\delta$ can be adjusted to zero and $s_{34} / S_{12}$ can be made negligible by using a sufficiently long absorbing vane.

This also explains why precision RVA's with sometimes much longer rotary sections do not necessarily exhibit smaller values of $\gamma$ [2], [3].

The slow frequency dependence of

$$
\frac{1}{2}\left[\gamma\left(90^{\circ}\right)+\gamma\left(270^{\circ}\right)\right]
$$

shows that the unwanted $\mathrm{TE}_{21}$ mode is efficiently attenuated due to the absorbing polyiron grooves. The values of

$$
\frac{1}{2}\left[\varepsilon\left(90^{\circ}\right)-\varepsilon\left(270^{\circ}\right)\right]
$$

and

$$
\frac{1}{2}\left[\gamma\left(90^{\circ}\right)-\gamma\left(270^{\circ}\right)\right]
$$

are partly due to uncertainty and indicate that the level of the unwanted $\mathrm{TM}_{01}$ mode is very small. Finally $\theta_{0}\left(90^{\circ}\right)-$ $\theta_{0}\left(270^{\circ}\right)$ is ascribed to excentricity of the worm gear.

$$
\overline{\bar{R}}=\left\{\begin{array}{lll}
r_{11} & R_{12} & r_{13} \\
R_{12} & r_{22} & r_{23} \\
r_{13} & r_{23} & r_{33}
\end{array}\right\}
$$

(a)

$\overline{\bar{s}}=\left\{\begin{array}{cccc}s_{11} & s_{12} & s_{13} & s_{14} \\ s_{12} & s_{22} & s_{23} & s_{24} \\ s_{13} & s_{23} & s_{33} & s_{34} \\ s_{14} & s_{24} & s_{34} & s_{44}\end{array}\right\}$

(b)

$$
\overline{\bar{T}}=\left\{\begin{array}{lll}
t_{11} & T_{12} & t_{13} \\
T_{12} & t_{22} & t_{23} \\
t_{13} & t_{23} & t_{33}
\end{array}\right\}
$$

(c)

Fig. 10. Scattering matrices of the three sections of the RVA. Orientations of coordinate systems are chosen parallel with the principal axes of the $\mathrm{TE}_{11}$ modes. (a) Input stator section. (b) Rotary section. (c) Output stator section.

\section{CONCLUSION}

A model for the rotary vane attenuator has been set up. It has been the aim to take all terms into account which are above the detection limit under high-sensitivity and highstability conditions. All the measurements of reflection and transmission parameters are very well explained by the model. The small systematic errors of the high-precision attenuation measurements in [3] are also in good agreement with the model.

By using the model with measured parameters, RVA's can be calibrated with high precision. The only errors left are due to limited readout accuracy, drift, and noise.

Methods are given for determination of the transmission parameters with the accuracy required for high-precision measurements. Even for this purpose, ordinary lowprecision instruments will suffice. A complete series of measurements may be performed without difficult and time-consuming tuning procedures. The procedures are, therefore, well suited for automatic computer-controlled apparatus and make multiple-frequency high-precision measurements practicable. Finally high-precision measurements may be extended to more than $200 \mathrm{GHz}$ where nonideal components have been the limiting factor up to now.

\section{APPENDIX I}

\section{DERIVATION OF THE RVA MODEL}

(Without CirCUlators)

The scattering matrix of the RVA is calculated by combining the scattering matrices $R, S$, and $T$ of the input stator section, the rotary section, and the output stator section of Fig. 10 by means of the rotation relations of Fig. 11. From 


$$
\left\{a_{R 2}, a_{R 2}, a_{S 1}, a_{S 4}\right\}=\left\{\begin{array}{cccc}
0 & 0 & \cos (0+\infty) & -\sin (0+\delta) \\
0 & 0 & \sin (\theta+\delta) & \cos (\theta+\delta) \\
\cos (\theta+\delta) & \sin (0+\delta) & 0 & 0 \\
-\sin (0+\delta) & \cos (0+\delta) & 0 & 0
\end{array}\right\}\left\{\begin{array}{l}
b_{R 2} \\
b_{R 3} \\
b_{S} \\
b_{S 4}
\end{array}\right\}
$$

(a)

$$
\left\{a_{\mathrm{T} 2}, a_{\mathrm{T} 2}, a_{\mathrm{S} 2}, a_{\mathrm{S} 3}\right\}=\left\{\begin{array}{cccc}
0 & 0 & \cos (0-\delta) & \sin (\theta-\delta) \\
0 & 0 & \sin (\theta-\delta) & \cos (0-\delta) \\
\cos (\theta-\delta) & \sin (\theta-\delta) & 0 & 0 \\
\sin (\theta-\delta) & \cos (0-\delta) & 0 & 0
\end{array}\right\}\left\{\begin{array}{l}
b_{\mathrm{T} 2} \\
b_{\mathrm{T} 3} \\
\mathrm{~b}_{\mathrm{S} 2} \\
\mathrm{~b}_{\mathrm{S} 4}
\end{array}\right\}
$$

(b)

Fig. 11. Matrices describing the rotation relations between fields in fixed and rotated coordinate systems. (a) Input rotary joint. (b) Output rotary joint.

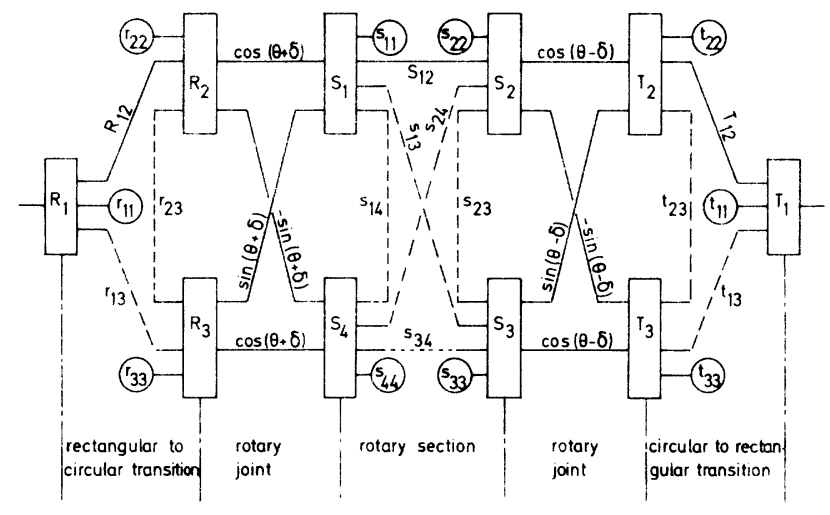

Fig. 12. Scattering diagram for the RVA

the rather complex structure of the RVA one can see that an exact calculation of the RVA scattering matrix yields a very large and probably useless expression. One is, therefore, forced to use approximate methods of calculation. It is easily seen that the RVA is ideal, i.e., follows the $\cos ^{2} \theta$ relationship, if all the small letter elements including $\delta$ are zero. In practice, all the small letter elements are considerably smaller than unity which makes a series development natural.

As an aid for setting up the series development the scattering diagram in Fig. 12 is used. ${ }^{1}$ All terms which are assumed to be above the limit of sensitivity are included. The series development contains a rather large number of terms which may be rearranged in the following way.

Reflection at port $R_{1}$ :

$$
\begin{aligned}
\rho_{R}= & a_{R}+b_{R} \sin ^{2} \theta+c_{R} \sin ^{2} 2 \theta+d_{R} \sin 2 \theta \\
& +e_{R} \sin 4 \theta .
\end{aligned}
$$

Reflection at port $T_{1}$ :

$$
\begin{aligned}
\rho_{T}= & a_{T}+b_{T} \sin ^{2} \theta+c_{T} \sin ^{2} 2 \theta+d_{T} \sin 2 \theta \\
& +e_{T} \sin 4 \theta .
\end{aligned}
$$

${ }^{1}$ The scattering diagram method is described in Appendix II.
Transmission between port $R_{1}$ and port $T_{1}$ :

$$
\begin{aligned}
T= & T_{0} \cos ^{2} \theta\left(1+\alpha \sin ^{2} \theta+\beta \sin ^{2} 2 \theta+j \varepsilon \tan \theta\right. \\
& \left.+\gamma \tan ^{2} \theta+\zeta \sin 2 \theta+\eta \sin 4 \theta\right) .
\end{aligned}
$$

The parameters of $\rho_{R}$ are given by

$$
\begin{aligned}
a_{R}= & r_{11}+R_{12}^{2} s_{11}+R_{12}^{2} S_{12}^{2} t_{22} \\
b_{R}= & R_{12}^{2}\left(s_{44}-s_{11}-S_{12}^{2} t_{22}\right) \\
c_{R}= & \frac{1}{4} R_{12}^{2} S_{12}^{2}\left(t_{33}-t_{22}\right) \\
d_{R}= & -R_{12}^{2}\left(\frac{1}{2} S_{12}^{2} t_{23}+s_{14}\right) \\
& +R_{12}\left\{r_{13}\left[s_{11}-s_{44}+\frac{1}{2} S_{12}^{2}\left(t_{22}+t_{33}\right)\right]\right. \\
& \left.+\frac{1}{2} R_{12} S_{12}\left[s_{13}\left(t_{22}-t_{33}\right)-s_{24}\left(t_{22}+t_{33}\right)\right]\right\} \\
e_{R}= & -\frac{1}{4} R_{12}^{2} S_{12}^{2} t_{23}+\frac{1}{4} R_{12} S_{12}\left(t_{22}-t_{33}\right) \\
& \cdot\left[r_{13} S_{12}+R_{12}\left(s_{13}-s_{24}\right)\right] .
\end{aligned}
$$

The parameters of $\rho_{T}$ are given by

$$
\begin{aligned}
a_{T}= & t_{11}+T_{12}^{2} s_{22}+T_{12}^{2} S_{12}^{2} r_{22} \\
b_{T}= & T_{12}^{2}\left(s_{33}-s_{22}-S_{12}^{2} r_{22}\right) \\
c_{T}= & \frac{1}{4} T_{12}^{2} S_{12}^{2}\left(r_{33}-r_{22}\right) \\
d_{T}= & T_{12}^{2}\left(s_{23}+\frac{1}{2} S_{12}^{2} r_{23}\right) \\
& -T_{12}\left\{t_{13}\left[s_{22}-s_{33}+\frac{1}{2} S_{12}^{2}\left(r_{22}+r_{33}\right)\right]\right. \\
& \left.-\frac{1}{2} T_{12} S_{12}\left[s_{24}\left(r_{22}-r_{33}\right)-s_{13}\left(r_{22}+r_{33}\right)\right]\right\} \\
e_{T}= & \frac{1}{4} T_{12}^{2} S_{12}^{2} r_{23}-\frac{1}{4} T_{12} S_{12}\left(r_{22}-r_{33}\right) \\
& \cdot\left[t_{13} S_{12}+T_{12}\left(s_{24}-s_{13}\right)\right] .
\end{aligned}
$$


The parameters of $T$ are given by

$$
\begin{aligned}
T_{0}= & R_{12} S_{12} T_{12}\left(1+s_{11} r_{22}+s_{22} t_{22}+S_{12}^{2} r_{22} t_{22}\right) \\
\alpha= & \left\{\left(s_{44}-s_{11}\right)\left(r_{22}-r_{33}\right)+\left(s_{33}-s_{22}\right)\left(t_{22}-t_{33}\right)\right. \\
& +\frac{1}{2} S_{12}^{2}\left[\left(r_{33}+r_{22}\right)\left(t_{33}-t_{22}\right)\right. \\
& \left.\left.+\left(t_{33}+t_{22}\right)\left(r_{33}-r_{22}\right)\right]\right\} \\
\beta= & -\frac{1}{4} S_{12}^{2}\left(r_{22}-r_{33}\right)\left(t_{22}-t_{33}\right) \\
j \varepsilon= & \frac{r_{13}}{R_{12}}+\frac{s_{13}-s_{24}}{S_{12}}-\frac{t_{13}}{T_{12}}-s_{14} r_{33} \\
& +r_{23} s_{44}-t_{23} s_{33}+s_{23} t_{33} \\
\gamma= & -\frac{s_{34}}{S_{12}}+\frac{r_{13} s_{13}}{R_{12} S_{12}}+\frac{t_{13} s_{24}}{T_{12} S_{12}}-\frac{r_{13} t_{13}}{R_{12} T_{12}} \\
& +\delta\left(\frac{r_{13}}{R_{12}}-\frac{s_{13}+s_{24}}{S_{12}}+\frac{t_{13}}{T_{12}}\right)-\delta^{2} \\
\zeta= & s_{14}\left(r_{33}-r_{22}\right)+s_{23}\left(t_{22}-t_{33}\right) \\
& +r_{23}\left[s_{11}-s_{44}+\frac{1}{2} S_{12}^{2}\left(t_{22}+t_{33}\right)\right] \\
& +t_{23}\left[s_{33}-s_{22}-\frac{1}{2} S_{12}^{2}\left(r_{22}+r_{33}\right)\right] \\
\eta= & \frac{1}{4} S_{12}^{2}\left[r_{23}\left(t_{22}-t_{33}\right)-t_{23}\left(r_{22}-r_{33}\right)\right] .
\end{aligned}
$$

It should be noted that the first-order terms in the expression for $j \varepsilon$ are purely imaginary due to the orientation of the coordinate systems. A real component in the expression for $j \varepsilon$ is equivalent to and cannot be distinguished from an error in the dial zero setting.

The large number of terms in the expressions above may give the impression of an overwhelming work of bookkeeping but this has not been so. For more complicated circuits one may prefer to develop computer methods for analytical calculations.

\section{APPENDIX II \\ Scattering Diagram Method for Calculating SCATtering Matrices of Microwave Circuits}

The scattering matrix of a composite circuit can be calculated by combining the known scattering matrices for the components entering the circuit [9]. This method becomes very complex for large circuits when all elements have to be taken into account. The scattering diagram method [10] leads to the same result by means of a series development which can be written down directly from the diagram. The only problem left is the bookkeeping of terms in the series development. The series development often converges rapidly because the components deviate only slightly from the ideal.

The ports of a specific component are represented by rectangular boxes with labels. The off-diagonal elements of the scattering matrix are represented by lines connecting the ports. The value of the scattering element is written on the line. In case of a nonreciprocal component, two scattering elements and two corresponding arrows for transmission direction are written on the line. Diagonal elements, i.e., reflections, are represented by circles with inscribed value connected to a port with a line. All lines representing scattering elements are connected to the same (inner) side of the port.

Connections between different components are represented by lines connected to the outer side of the ports. Arbitrary complex transmission factors may be written on these lines.

An element of the scattering matrix for the composite circuit can be calculated by summing up the infinite number of contributions from the different paths of signal flow.

The following rules for signal flow should be observed.

1) A signal traveling along a line is multiplied by the expression written on the line.

2) A signal reaching a port from one side can leave the port along any of the lines connected to the other side of the port.

3) All possible signal paths should be taken into account. If a path contains a loop which can be followed in both directions, the contributions of each should be taken into account.

\section{ACKNOWLEDGMENT}

The author wishes to thank his wife Birthe for a careful and independent check of the analytical calculations and for the computer programming. He wishes also to thank L. D. Nielsen for many valuable comments during the preparation of this paper.

\section{REFERENCES}

[1] G. F. Engen and R. W. Beatty, "Microwave attenuation measurements with accuracies from 0.0001 to 0.06 decibel over a range of 0.01 to 50 decibels," J. Res. Nat. Bur. Stand ., vol. 64C (Eng. and Instrum.), no. 2, pp. 139-145, Apr.-June 1960.

[2] W. E. Little, W. Larson, and B. J. Kinder, "Rotary vane attenuator with an optical readout," J. Res. Nat. Bur. Stand., vol. 75C, no. 1, pp. 1-5, Jan.-Mar. 1971.

[3] F. L. Warner, D. O. Watton, and P. Herman, "A very accurate $\mathrm{X}$-band rotary attenuator with an absolute digital angular measuring system," IEEE Trans. Instrum. Meas., vol. IM-21, pp. 446-450, 1972.

[4] F. L. Warner, D. O. Watton, P. Herman, and P. Cummings, "Automatic calibration of rotary-vane attenuators on a modulated subcarrier system," IEEE Trans. Instrum. Meas., vol. IM-25, pp. 409-413, 1976.

[5] F. L. Warner, "Microwave attenuation measurements," IEE Monograph Ser. 19. Stevenage, England: Peregrinus.

[6] B. P. Hand, "Broadband rotary waveguide attenuator," Electronics, vol. 27, pp. 184-185, Jan. 1954.

[7] J. D. Holm et al., "Reflections from rotary-vane precision attenuators," IEEE Trans. Microwave Theory Tech., vol. MTT-15, pp. 123124,1967

[8] T. Y. Otoshi and C. T. Stelzried, "A precision compact rotary vane attenuator," IEEE Trans. Microwave Theory Tech., vol. MTT-19, pp. $843-854,1971$

[9] T. A. Abele, "Über die Streumatrix Allgemein Zusammengeschalteter Mehrpole" ("The scattering matrix of a general interconnection of multipoles"), Arch. Elek. Übertragung, vol. 14, pt. 6, 1960, pp. 262-268. (Transl. in NASA Rep. NASA-CR-101404.)

[10] D. J. M. Park, "Scattering diagrams and some new nonreciprocal waveguide circuits," Microwave J., vol. 6, no. 6, pp. 65-79, June 1963. 